International Mathematical Forum, 2, 2007, no. 52, 2587 - 2591

\title{
A Vector Lyapunov Approach to the Stability Problem for the n-Population Continuous Time Replicator Dynamics
}

\author{
Zvi Retchkiman Königsberg \\ Instituto Politécnico Nacional, CIC \\ Mineria 17-2, Col. Escandon, Mexico D.F 11800, Mexico \\ mzvi@cic.ipn.mx
}

\begin{abstract}
In this paper the stability problem for the n-population continuous time replicator dynamics using vector Lyapunov methods is addressed. After introducing the evolutionary stable strategy concept and proving that it is equivalent to being a strict Nash equilibrium, the n-population continuous time replicator dynamics equation is presented. Finally, it is shown that every strict Nash equilibrium is asymptotically stable in the associated dynamics via Lyapunov methods.
\end{abstract}

Mathematics Subject Classification: 91A22, 34D20, 93D05

\section{Preliminaries}

Definition 1.1 A continuous function $\varphi: \Re_{+} \rightarrow \Re_{+}$is said to belong to class $\mathcal{K}$ if it is strictly increasing, $\alpha(0)=0$ and $\alpha(r) \rightarrow \infty$ as $r \rightarrow \infty$.

Consider the differential system

$$
d x / d t=f(t, x)
$$

where $f: C\left(\Re_{+} \times \Re^{n}\right) \rightarrow \Re^{n}\left(x\left(t, t_{0}\right)=x_{0}\right)$. Suppose that $f$ is smooth enough to guarantee existence, uniqueness and continuous dependence of solutions $x(t)=x\left(t, t_{0}, x_{0}\right)$ of system 1 . Then, the next result takes care of its stability.

Theorem 1.2 [1] Let $V: \Re_{+} \times B(\rho) \subset \Re^{n} \rightarrow \Re_{+}^{s}\left(B(\rho)=\left\{x \in \Re^{n}:\|x\|<\right.\right.$ $\rho\})$ be a continuously differentiable vector Lyapunov function, such that 


$$
V_{0}(t, x)=\sum_{i=1}^{s} V_{i}(t, x)
$$

satisfies

$$
\varphi_{1}\left(\|x\|_{2}\right) \leq V_{0}(t, x) \leq \varphi_{2}\left(\|x\|_{2}\right)
$$

for $\varphi_{1}, \varphi_{2} \in K$ and, the differential inequality

$$
\dot{V}((x, t) \leq h[t, V(x, t)]
$$

holds for all $(t, x) \in \Re_{+} \times B(\rho) \subset \Re^{n}$ where $h: \Re_{+} \times \Re_{+}^{s} \rightarrow \Re^{s}$ is a continuously differentiable function such that $h(t, 0) \equiv 0$ and $h(t, V)$ is quasimonotone nondecreasing in $V$ (for all $V \in \Re_{+}^{s}$ and $t \in \Re_{+}$). Let $\omega\left(t ; w_{0}, t_{0}\right)$ be the solution of the comparison system

$$
\dot{\omega}(t)=h(\omega(t), t), \omega\left(t_{0}\right)=\omega_{0} \geq 0 .
$$

Then,

$$
\dot{V}(t, x(t)) \leq \omega\left(t ; \omega_{0}, t_{0}\right), t \geq t_{0}
$$

provided that $V\left(t_{0}, x_{0}\right) \leq \omega_{0}$. Therefore, the stability properties of the trivial solution $\omega=0$ of the comparison system imply the corresponding stability properties of $x$.

Corollary 1.3 In Theorem (1.2):

i). If $h(t, \omega) \equiv 0$ we get stability.

ii). If $h(t, \omega)=-\alpha \omega, \alpha>0$, we get asymptotic stability.

Remark 1.4 We encorage those readers not familiar with game theory, its basic concepts and mathematical notations, to see [2] and [3].

Definition 1.5 Let $\Theta$ be the polyhedron of mixed strategies profiles, a strategy profile $x \in \Theta$ is said to be a strict Nash equilibrium if $\{x\}=B(x)$ the best reply function.

Definition 1.6 Let $u_{i}: \Theta \rightarrow \Re_{+}, i \in I=1,2, \ldots n$ be the utility function. A strategy profile $x \in \Theta$ is evolutionary stable if for every strategy $y \neq x$ there exists some $\epsilon_{y} \in(0,1)$ such that for all $\epsilon \in\left(0, \epsilon_{y}\right)$ and with $w=\epsilon y+(1-\epsilon) x$ 


$$
u_{i}\left(x_{i}, w_{-i}\right)>u_{i}\left(y_{i}, w_{-i}\right) \text { for some } i \in I
$$

The next result's proof is much in the flavor of the one provided in [2].

Proposition 1.7 A strategy profile $x \in \Theta$ is evolutionary stable if and only if $x$ is a strict Nash equilibrium.

Proof 1.8 First assume that $x$ is a strict Nash equilibrium then $u_{i}\left(x_{i}, x_{-i}\right)>$ $u_{i}\left(y_{i}, x_{-i}\right)$ for every $i$, by continuity taking $\epsilon$ small we get that $u_{i}(x_{i}, \underbrace{\epsilon y_{-i}+(1-\epsilon) x_{-i}}_{w_{-i}})>u_{i}(y_{i}, \underbrace{\epsilon y_{-i}+(1-\epsilon) x_{-i}}_{w_{-i}})$.

Now let us prove the converse: Take $i \in I$ arbitrary and $y_{i} \in B_{i}(x), y_{i} \neq$ $x_{i} \Rightarrow u_{i}\left(y_{i}, x_{-i}\right)>u_{i}\left(x_{i}, x_{-i}\right)$ and set $y_{j}=x_{j}$ for $j \neq i$ (i.e., $y_{i} \in B_{i}(x)$ for all $i \in I \Rightarrow u_{i}\left(y_{i}, x_{-i}\right)>u_{i}\left(x_{i}, x_{-i}\right)$ for all $\left.i \in I(\vee)\right)$. Since $x \in \Theta$ is evolutionary stable, for some $i \in I$

$$
\begin{gathered}
\epsilon u_{i}\left(x_{i}, y_{-i}\right)+(1-\epsilon) u_{i}\left(x_{i}, x_{-i}\right) \\
>\epsilon u_{i}\left(y_{i}, y_{-i}\right)+(1-\epsilon) u_{i}\left(y_{i}, x_{-i}\right) \stackrel{(\bullet)}{\geq} \epsilon u_{i}\left(y_{i}, y_{-i}\right)+(1-\epsilon) u_{i}\left(x_{i}, x_{-i}\right) \\
\Rightarrow u_{i}\left(x_{i}, y_{-i}\right) \geq u_{i}\left(y_{i}, y_{-i}\right)
\end{gathered}
$$

i.e., $x_{i} \in B_{i}(y)$ for some $i$ but $y_{i} \in B_{i}(x)$ for all $i$ therefore, $y_{i}=x_{i}$ is a Nash equilibrium and $\left\{x_{i}\right\}=B_{i}(x)$ and since $i \in I=\{1,2 \ldots, n\}$ was arbitrary we conclude that $\{x\}=B(x)$ and therefore $x$ is a strict Nash equilibrium.

Next, the n-population continuous time replicator dynamics is presented. Unlike the single population setting, there are two versions of the continuous time n-population replicator dynamics. We will deal with the one suggested by Taylor [4] which has the form:

$$
\dot{x}_{i h}=\left[u_{i}\left(e_{i}^{h}, x_{-i}\right)-u_{i}(x)\right] x_{i}
$$

with $x \in \Theta$, pure strategy $h$ and $i \in I=\{1,2, \ldots n\}$, which by standard Lipshitz arguments has a unique solution. 


\section{Main Result}

Theorem 2.1 Every $x \in \Theta$, strict Nash equilibrium is asymptotically stable in the n-population continuous time replicator dynamics

Proof 2.2 Let $x \in \Theta$, be a strict Nash equilibrium then, $x$ is a vertex of $\Theta$ i.e., $x_{i}=e_{i}^{h_{i}}$ and $u_{i}\left(e_{i}^{h_{i}}, x_{-i}\right)>u_{i}\left(z_{i}, x_{-i}\right)$ for all $i \in I=\{1,2, \ldots n\}$ and $z_{i} \neq x_{i}$. By continuity this implies that $u_{i}\left(y_{i}, y_{-i}\right)>u_{i}\left(z_{i}, y_{-i}\right)$ in a $N(x) \cap \Theta$. Now take the neighborhood as small as needed in such away that it contains no other vertex of $\Theta$ i.e.., the set of strategies $y_{-i}$ is included in the set of strategies $x_{-i}$. Therefore, from the definition of strict Nash equilibrium with the $\left.x_{-i}\right|_{y_{-i}}$, we get that $u_{i}\left(e_{i}^{h_{i}}, y_{-i}\right)>u_{i}\left(y_{i}, y_{-i}\right)=u_{i}(y)$. for all $y \neq x$, $y \in N(x) \cap \Theta$ and all $i \in I$. Now, define as our vector Lyapunov function $V(y)=\left[V_{1}(y), V_{2}(y), \ldots, V_{n}(y)\right]^{T} ;$ where $V_{i}(y)=\sum_{i} x_{i} \log \frac{x_{i}}{y_{i}}, 1 \leq i \leq n$ are relative entropy functions defined in a specific neighborhood of $x$ which without loss of generality will be taken equal to $N(x)$. Then, applying theorem 1.2 in $N(x) \cap \Theta$, we can verify that all the its conditions are satisfied and that,

$$
\dot{V}(y)=-\left[u_{1}\left(e_{1}^{h_{1}}-y, y_{-1}\right), u_{2}\left(e_{2}^{h_{2}}-y, y_{-2}\right), \ldots, u_{|I|}\left(e_{n}^{h_{n}}-y, y_{-n}\right)\right]^{T}<0^{T}
$$

implying that $x$ is asymptotically stable in the $n$-population continuous time replicator dynamics.

Remark 2.3 Converse of (2.1) is also true [2]. Therefore, being a strictly Nash equilibrium is equivalent to asymptotic stability.

\section{Conclusions}

Asymptotic stability for the continuous time n-population replicator dynamics was shown to hold, in a more natural way than other previous scalar approaches by means of employing vector Lyapunov functions.

\section{References}

[1] V. Lakshmikantham, V. M. Matrosov and S. Sivasundaram, Vector Lyapunov Functions and Stability Analysis of Nonlinear Systems, Kluwer Academic Publ., Dordrecht, 1991.

[2] J. W. Weibull, Evolutionary Game Theory, MIT Press 1997.

[3] M. J. Osborne and A. Rubinstein, A Course in Game Theory, MIT Press 1994. 
[4] P. Taylor, Evolutionary stable strategies with two types of players, Journal of Applied Probability 1979, 16: 76-83.

Received: April 3, 2007 